IBIMA Publishing

International Journal of Case Reports in Medicine

http://www.ibimapublishing.com/journals/IJCRM/ijcrm.html

ijcrm.html Vol. 2016 (2016), Article ID 832328, 6 pages

DOI: $10.5171 / 2016.832328$

Case Report

\title{
Eosinophilic Pustular Folliculitis in a Patient with Behçet's Disease
}

\author{
Min Ju Choi, Sung Bin Cho, Suhyun Cho, Do Young Kim and Dongsik Bang \\ Department of Dermatology and Cutaneous Biology Research Institute, Yonsei University College of \\ Medicine, Seoul, Korea \\ Correspondence should be addressed to: Dongsik Bang; dbang@yuhs.ac \\ Received date: 27 January 2014; Accepted date: 6 October 2014; \\ Published date: 19 September 2016 \\ Academic Editor: Valeria Brazzelli \\ Copyright (C) 2016. Min Ju Choi, Sung Bin Cho, Suhyun Cho, Do Young Kim and Dongsik Bang. \\ Distributed under Creative Commons CC-BY 4.0
}

\begin{abstract}
Behçet's disease (BD) is a chronic multisystemic inflammatory disease with chronic wax and waning course. Eosinophilic pustular folliculitis (EPF) is characterized by pruritic follicular papules and pustules that may involve the face, trunk, and upper arms. The precise etiopathogenesis of EPF is not yet elucidated. Immunological aberrations have been suggested to play pivotal roles, as the most common type of EPF is associated with HIV infection. Herein, we describe a patient with BD who presented with multiple vesicopustular lesions that were pathologically proven to represent EPF, and further postulate that EPF may be considered as cutaneous lesions of $\mathrm{BD}$, although further studies are necessitated.
\end{abstract}

Keywords: Behçet's disease, Eosinophilic pustular folliculitis, Pustule

\section{Introduction}

Behçet's disease (BD) is a chronic

inflammatory disease which is

characterized by recurrent oral aphthae, genital ulcers, ocular lesions, and cutaneous lesions. Cutaneous lesions of BD can manifest as erythema nodosum-like lesions, pseudofolliculitis, papulopustular eruptions, erythema multiform-like lesions, Sweet's syndrome-like lesions, and subcutaneous thrombophlebitis (1-3). The pathogenesis of BD is complex, and genetic 
susceptibility, infection, and various cytokines are thought to play a role. Traditionally, Th1 mediated pathway is thought to be upregulated in $\mathrm{BD}$, resulting in increased IFN- $\gamma$ and IL-12 levels. Recently, researchers have proposed that Th17 cytokines such as IL-17 and IL-23 may also play a part in the pathogenesis of BD $(4,5)$. Among various skin lesions, until now, there has been no report of eosinophilic pustular folliculitis (EPF) in association with BD. In this report, we report a patient with Behçet's disease who presented with multiple vesicopustular lesions that were pathologically proven as EPF.

\section{Case Report}

A 45-year-old Korean woman presented to our department with multiple itchy erythematous papules on the face, anterior chest, upper back, and right upper arm that had progressed over a one-year period. The patient had been treated at private clinics with systemic antibiotics such as cephalosporin and minocycline, as well as systemic antiviral agents such as acyclovir and famciclovir. However, her skin symptoms were poorly controlled. Her medical history indicated that she had been treated for pulmonary tuberculosis 30 years prior. In addition, she had a history of recurrent oral ulcerations that had persisted for over ten years and genital ulcerations that had persisted for over six years.

On her initial visit, multiple vesicopustular erythematous papules were noted on the patient's face, anterior chest, upper back, and right upper arm (Figure 1). Laboratory tests revealed an elevated erythrocyte sedimentation rate of $65 \mathrm{~mm} /$ hour (normal range, $\leq 20 \mathrm{~mm} /$ hour). The results of other tests, including serologic test for human immunodeficiency virus (HIV), anticyclic citrullinated peptide antibodies, and HLA B51, were negative. Pathergy tests were performed, and pustule formation was observed within 48 hours at the injection sites. Computed tomography of the chest, abdomen, and pelvis did not demonstrate any findings suggestive of lymphoproliferative disease or internal malignancies. 


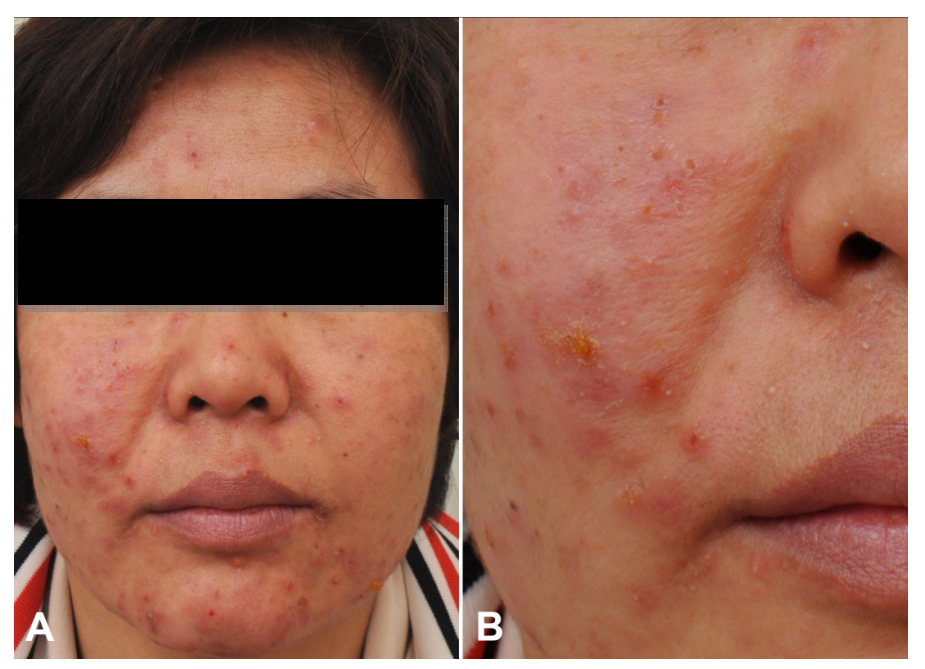

Figure 1: (A and B) Multiple erythematous vesicopustular papules with oozing crusts on the face

Specimens obtained from the patient's facial skin lesions showed marked infiltration of eosinophils into the epidermis, hair follicles, and perifollicular areas. There was also lymphocytic infiltration in the dermis near the vessels (Figure 2). Direct immunofluorescence studies of the biopsy specimens were negative. The patient was diagnosed with BD based on the guidelines of the International Study Group for BD with diagnostic criteria of recurrent oral ulceration, genital ulceration and positive pathergy reaction (6). She was also diagnosed with EPF based on the histopathology and clinical manifestations (7). The patient showed clinical improvements in the vesicopustular skin lesions with the systemic administration of cyclosporine and topical $0.1 \%$ tacrolimus ointment (Protopic ${ }^{\circledR}$; Astellas Toyama Co., Ltd., Toyama, Japan). 


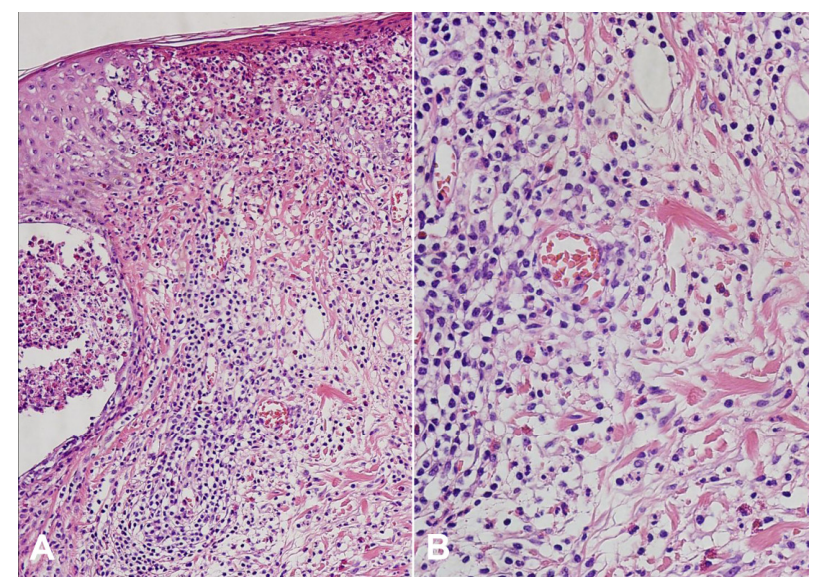

Figure 2: (A) Marked infiltration of eosinophils into hair follicles and perifollicular areas with (B) dermal lymphocytic infiltration around the blood vessels

(H\&E, A: X200, B: X400)

\section{Discussion}

EPF is characterized by pruritic follicular papules and pustules and may involve the face, trunk, and upper arms (7). There are three clinical types of EPF: (i) classic EPF, which typically occurs in Japanese patients with a seborrheic distribution; (ii) EPF associated with immunosuppression, which mainly occurs in patients with HIV infections or hematologic or lymphoproliferative disease; and (iii) EPF of infancy or the neonatal period (7). The precise etiopathogenesis of EPF is not yet explained, however, immunological alterations have been suggested to play pivotal roles (8).

EPF can occur in patients experiencing HIVnegative immunosuppression, including chronic lymphocytic lymphoma, nonHodgkin's lymphoma, anaplastic anemia, and bone marrow transplantation $(8,9)$. In the present case, serologic analysis was negative for HIV and peripheral blood cell test and computed tomography did not demonstrate any relevant hematologic or lymphoproliferative diseases.

Papulopustular lesions, pseudofolliculitis, and acneiform nodules are commonly observed as cutaneous manifestations of BD, especially in male patients $(1,10)$. However, such lesions are nonspecific and are difficult to clinically differentiate from other diseases, such as folliculitis or acne vulgaris. Recent reports have demonstrated that histopathogical findings of vasculitis in papulopustular lesions may be regarded as significant diagnostic criteria in patients for whom diagnoses of BD are suspected (10, 11). Our patient also presented with findings that were suggestive of lymphocytic vasculitis.

The first line of treatment in EPF is usually topical steroids and oral indomethacin which are responsive in approximately $90 \%$ of patients (12). Nakahigashi K. et al discovered that prostaglandin (PG) such as PGD2/PGJ2 induced eotaxin-3 and resulted 
in eosinophil infiltration in EPF, and postulated that indomethacin, a COX inhibitor, would downregulate the synthesis of PG and lead to therapy in EPF (13). Patients with EPF who are resistant to classical treatments are treated with various therapies such as minocycline, doxycycline, dapsone, cyclosporine, and topical tacrolimus $(12,14)$. Our patient was responsive to oral cyclosporine and topical tacrolimus ointment that resulted in clearance of the facial EPF lesions.

The patient in this case presented with histopathologic features that are both suggestive of EPF and skin manifestations in BD. Numerous eosinophils were gathered around the follicles and lymphohistiocyte infiltration around the vessels were noted.

The etiopathogenetic significance of eosinophils in papulopustular skin lesions in BD patients has not been fully investigated. Therefore, it is unclear whether EPF should be regarded as a variant of papulopustular lesions in $\mathrm{BD}$ or whether $\mathrm{BD}$ should be regarded as an initiating or aggravating factor of EPF in our patient. Further studies including larger samples of BD patients and histopathological reviews of papulopustular lesions are necessary to confirm our findings.

\section{References}

1. Sakane T, Takeno M, Suzuki N, and Inaba G. (1999) "Behçet's disease," N Engl J Med. 341(17):1284-91.
2. Lee ES, Bang D, and Lee S. (1997) "Dermatologic manifestation of Behçet's disease," Yonsei Med J. 38(6):380-9.

3. Kurokawa MS, Yoshikawa H, and Suzuki N. (2004) “Behçet's disease," Semin Respir Crit Care Med. 25(5):557-8.

4. Ben Ahmed M, Houman H, Miled M, Dellagi K, and Louzir H. (2004) "Involvement of chemokines and Th1 cytokines in the pathogenesis of mucocutaneous lesions of Behcet's disease." Arthritis Rheum. 50(7):2291-5.

5. Lew W, Chang JY, Jung JY, and Bang D. (2008) "Increased expression of interleukin23 p19 mRNA in erythema nodosum-like lesions of Behcet's disease," Br J Dermatol. 158(3):505-11.

6. (1990) "International study group for Behçet's disease. Criteria for diagnosis of Behçet's disease," Lancet. 335(8697):107880.

7. Moritz DL and Elmets CA. (1991) "Eosinophilic pustular folliculitis," J Am Acad Dermatol. 24(5):903-7.

8. Ogawa E, Okuyama R, Niizuma H, Tagami H, Tsuchiya S, and Aiba S. (2009) "Eosinophilic pustular folliculitis occurring after bone marrow transplantation in a child with aplastic anaemia," Acta Derm Venereol. 89(2):200-1.

9. Scavo S, Magro G, and Caltabiano R. (2010)

"Erythematous and edematous eruption of the face," Int J Dermatol. 49(9):975-7.

10. Kalkan G, Karadag AS, Astarci HM, Akbay G, Ustun H, and Eksioglu M. (2009) A histopathological approach: when 
papulopustular lesions should be in the diagnostic criteria of Behçet's disease?," J Eur Acad Dermatol Venereol. 23(9):1056-60.

11. Ilknur T, Pabuççuoglu U, Akin C, Lebe B, and Gunes AT. (2006) "Histopathologic and direct immunofluorescence findings of the papulopustular lesions in Behçet's disease," Eur J Dermatol. 16(2):146-50.

12. Schmieder A, Damm F, Haus G, Lentz K, Goerdt S, and Peitsch WK. (2013) "Extended eosinophilic pustular folliculitis with reactive bone marrow hypereosinophilia responsive to combined treatment with indomethacin, dapsone, and narrow-band UVB phototherapy," J Am Acad Dermatol. 69(6):312-4.

13. Nakahigashi K1, Doi $H$, Otsuka A, Hirabayashi T, Murakami M, Urade Y, Zouboulis CC, Tanizaki H, Egawa G, Miyachi Y, and Kabashima K. (2012) "PGD2 induces eotaxin-3 via PPAR $\gamma$ from sebocytes: a possible pathogenesis of eosinophilic pustular folliculitis," J Allergy Clin Immunol. $129(2): 536-43$.

14. Ng SS and Tay YK. (2012) "Successful treatment of eosinophilic pustular folliculitis with topical tacrolimus 0.1 percent ointment," Dermatol Online J. 18(2):10. 